CARNETS DE Carnets de géographes

GÉOGRAPHES.

$8 \mid 2015$

Géographie(s) de la lenteur

L'informalité dans le quartier de la Goutte d'Or à Paris

Économie immigrante, africanité et politiques urbaines

Milly Aoustin-Sow

2 OpenEdition

Journals

Édition électronique

URL : http://journals.openedition.org/cdg/311

DOI : $10.4000 /$ cdg. 311

ISSN : 2107-7266

Éditeur

UMR 245 - CESSMA

Référence électronique

Milly Aoustin-Sow, "L'informalité dans le quartier de la Goutte d'Or à Paris », Carnets de géographes [En ligne], 8 | 2015, mis en ligne le 01 septembre 2015, consulté le 23 septembre 2020. URL : http:// journals.openedition.org/cdg/311 ; DOI : https://doi.org/10.4000/cdg.311

Ce document a été généré automatiquement le 23 septembre 2020.

\title{
(c) ()९
}

La revue Carnets de géographes est mise à disposition selon les termes de la Licence Creative Commons Attribution - Pas d'Utilisation Commerciale - Pas de Modification 4.0 International. 


\title{
L'informalité dans le quartier de la Goutte d'Or à Paris
}

Économie immigrante, africanité et politiques urbaines

\author{
Milly Aoustin-Sow
}

1 Dans le $18^{\text {ème }}$ arrondissement de Paris, le quartier populaire et immigrant de la Goutte d'Or accueille de nombreux commerces tenus par des populations d'origine étrangère (africaine, asiatique, européenne) proposant un large choix de produits dits exotiques et de services aux immigrants. Par conséquent, il attire une clientèle cosmopolite et hétérogène ainsi que des badauds venus du quartier mais également de toute l'île-deFrance, voire de l'étranger. La Goutte d'Or est à ce titre le quartier parisien qui possède le rapport commerce/habitant le plus élevé de la capitale et le plus grand nombre de commerces en activité en valeur absolue.

Depuis les années soixante-dix, l'Afrique règne, les Africains s'affichent sans complexe et les affaires vont bon train. L'habit ne fait pas le moine pourrait être la devise un soupçon provocatrice de cette portion de territoire connue davantage pour les fidèles musulmans dont les prières s'étendent jusque sur la chaussée et les trafics de rue en tout genre que pour le chiffre d'affaires généré par l'activité commerciale de la rue Dejean et des boutiques alentours.

3 Car simultanément, en marge des commerces établis, il est aisé de remarquer des vendeurs et vendeuses à la sauvette aux abords des stations de métro BarbèsRochechouart et Château-Rouge, Marcadet dans une moindre mesure, eux aussi apparemment d'origine étrangère. En effet, qui n'a jamais été interpelé en sortant du métro Barbès par des hommes murmurant « Marlboro, Marlboro, Marlboro!» signifiant qu'ils revendent des paquets de cigarettes américaines au rabais, ou par des femmes en boubou qui vendent des épis de maïs grillés. Les exemples ne manquent pas. Cependant, le commerce informel à la Goutte d'or ne se limite pas aux ventes à la sauvette dans la rue qui restent très localisées, il a également lieu dans les boutiques, les logements et les interstices multiples du quartier. Qu'il s'agisse de vente ambulante dans la rue ou de services marchands à domicile plus ou moins légaux, ce type de d'activités assez caractéristiques se regroupe communément sous le nom générique d' 
économie informelle, d'où un amalgame facile entre immigration et informalité. Formel et informel coexistent et interagissent, les frontières n'étant pas catégoriques et aisées.

4 En réalité, depuis sa constitution, la Goutte d'Or est marquée par une économie immigrante puissante qui y prend source avec le lot d'activités urbaines informelles qui en découlent et y prospèrent. Cumulant les difficultés économiques et sociales liées aux caractéristiques de la population qui le compose, le quartier est inscrit dans les dispositifs de politique de la ville depuis 1984. Cette thèse a ainsi eu pour objectif de restituer l'informalité de la Goutte d'Or dans son intégralité, d'en analyser les modalités sociétales et économiques pour en révéler les impacts et les enjeux territoriaux et urbains. La présence de pratiques informelles dans la capitale française a suscité d'emblée une question centrale : quels sont la place, le rôle et l'avenir de ces pratiques dans la ville ? L'agencement de la réflexion s'est articulé selon trois axes : théorique et contextuel; géographique et économique; politique.

L'informalité revêt une certaine complexité dans la mesure où a priori aucun des secteurs de la vie n'est épargné. Au départ, l'informalité étudiée ne visait que les commerces et la sphère économique. Or, très rapidement, l'informalité s'est étendue et imposée à l'ensemble du quartier, ce qui a posé problème pour définir une méthodologie claire puisque les contours de l'informalité au sein du quartier sont restés flous, parfois jusqu'au bout de la recherche. Il a alors fallu s'en accommoder.

La Goutte d'Or n'excède pas les 27 hectares mais la diversité et la densité de ses commerces, des produits, des services, des langues, des cultures, des pratiques, des groupes aux intérêts divers qui coexistent sur ce morceau de territoire étriqué est conséquente et explique la difficulté de collectes de matériaux cohérents. Une pratique quotidienne du terrain, bi-hebdomadaire par la suite, des relevés des changements, des évènements, des prises de photographie, de présence aux réunions de quartier, des entretiens informels la plupart du temps.. ont complété l'étude. Aussi, une approche globale de l'informalité a-t-elle été privilégiée au détriment d'une connaissance fine d'un groupe spécifique ou d'une activité particulière, car cela était de toute évidence difficilement réalisable dans le cadre d'un travail individuel. Un recensement des activités informelles le plus exhaustif possible a été établi. En outre, le terrain d'étude a été découpé en trois secteurs (Marcadet au Nord, Château-Rouge au centre et Barbès au Sud) renvoyant à des réalités commerciales et sociétales distinctes.

7 Parmi les résultats de cette thèse, trois aspects essentiels sont à retenir. Le premier se situe au niveau de l'ancrage spatio-temporel profond de l'informalité. Un ancrage historique tout d'abord qui remonte au moins à la fin du $18^{\text {ème }}$ siècle (Zalc, Bruno, Blanchard, Spire) et fait du coup voler en éclats les théories douteuses associant l'arrivée des immigrants étrangers au développement de l'informalité. Ensuite, un ancrage économique marqué de l'informalité dans lequel les commerces occupent une place significative. Le nombre et la densité de l'activité économique (commerces \& artisanat) du quartier associée à l'intensité des activités donnent une position stratégique à la Goutte d'Or sur l'échiquier métropolitain.

8 Le deuxième aspect a trait au changement de dimension de l'informalité. Désormais, celle-ci ne se limite plus à l'espace du commerce et de l'artisanat mais s'étend à l'ensemble du quartier. L'informalité commerciale initiale opère un basculement vers une informalité territoriale du quartier, la Goutte d'Or s'érigeant alors en véritable hub africain. La centralité immigrée de Toubon \& Messamah devient une «centralité circulatoire », incontournable dans l'économie francilienne. 
Le troisième aspect tient dans les politiques urbaines successives visant un changement dans la composante sociologique du quartier. Elles n'ont pas suffi à éradiquer les activités informelles malgré différents postulats (Toubon \& Messamah, 1990, APUR, Mairie de Paris...) car au vu de ce qui s'y observe et s'y pratique encore de nos jours, les activités informelles dans le quartier de la Goutte d'Or continuent bel et bien. Les acteurs ne semblent pas avoir compris l'importance que l'informalité revêt dans le quartier. Grosso modo, son appréhension se limite à l'espace public et aux nuisances générées, ce qui compromet une approche renouvelée de l'informalité. Car, plus que l'aspect commercial et l'aspect territorial, c'est la dimension immatérielle de l'informalité qu'il s'agit de saisir à travers l'africanité du quartier. Sans la prise en compte de l'immatérialité de la Goutte d'Or, de cet esprit des lieux (Prats \& Thibault, 2003 ; Turgeon, 2008), il est à supposer que les métamorphoses urbaines espérées par certains (Mairie de Paris) soient, pour le moment, vouées à l'échec.

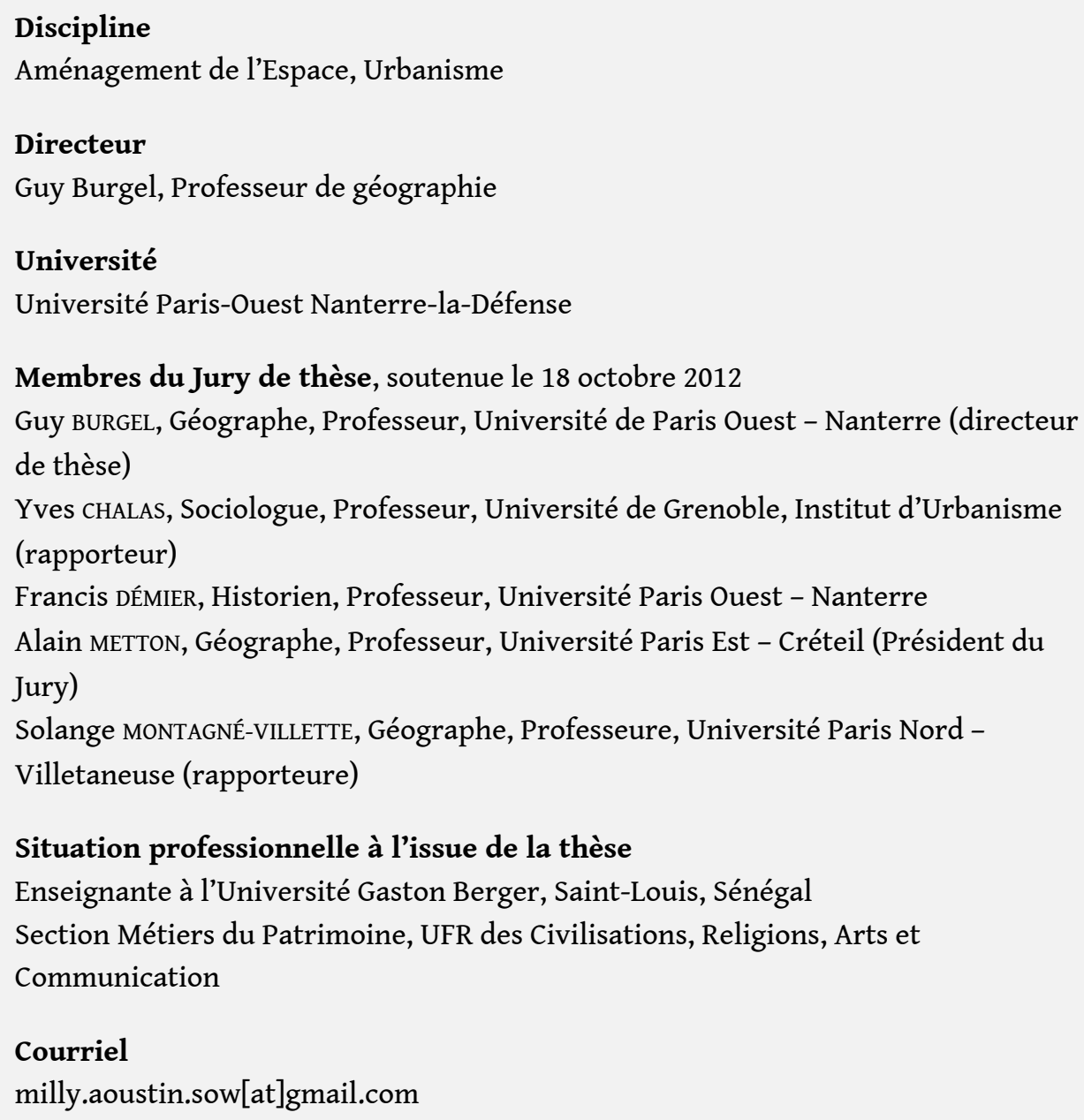


INDEX

Thèmes : Carnets de soutenances 